\title{
Insight into the relationship between obesity-induced low-level chronic inflammation and COVID-19 infection
}

\author{
Jihye Kim $\mathbb{D}^{1} \cdot$ Jae-Hwan $\mathrm{Nam}^{2}$
}

Received: 3 May 2020 / Revised: 10 May 2020 / Accepted: 12 May 2020 / Published online: 22 May 2020

(c) Springer Nature Limited 2020

Coronavirus disease (COVID-19), caused by severe acute respiratory syndrome coronavirus 2 (SARS-CoV-2), has rapidly caused a pandemic. Recent reports showed that individuals with obesity may be more vulnerable to severe complications of COVID-19 by virtue of the increased risk of chronic diseases driven by obesity [1]. The reasons for this are not well known, but chronic inflammation is suspected to be one of the causes. Obesity is a state of chronic low-grade systemic inflammation, which is characterized by increased proinflammatory cytokine secretion from adipose tissue and the infiltration of leukocytes, including macrophages, into the adipose tissue. Thus, this chronic inflammation impairs insulin signaling in adipocytes, causing insulin resistance, and contributing to the development of metabolic disorders such as cardiovascular disease, type 2 diabetes, and hypertension [2], which are well known comorbidities that adversely affect the outcome of patients with COVID-19.

In addition, patients with severe COVID-19 commonly show cytokine storms, which refer to an excessive and uncontrolled release of proinflammatory cytokines such as tumor necrosis factor- $\alpha$, monocyte chemotactic protein-1, and interleukin-6 (IL-6). In particular, serum IL-6 levels in those with severe COVID-19 were significantly higher than in those with mild cases $[1,3]$. Although most people with COVID-19 develop no symptoms or have only mild illness, the evidence shows that about $14 \%$ of COVID-19 patients develop severe symptoms requiring hospitalization and oxygen support, while 5\% develop acute respiratory distress

Jae-Hwan Nam

jhnam@catholic.ac.kr

1 Department of Medical Nutrition, Graduate School of East-West Medical Science, Kyung Hee University, Yongin 17104, Republic of Korea

2 Department of Biotechnology, The Catholic University of Korea, Bucheon 14662, Republic of Korea syndrome, sepsis, septic shock, and multiorgan failure $[4,5]$, and these are all related to inflammatory responses.

Individuals living with obesity have chronically higher leptin and lower adiponectin concentrations. This unfavorable hormone status leads to a dysregulation of the immune response and can contribute to the pathogenesis of obesityrelated complications [6]. In the basal state, individuals with obesity have a higher concentration of proinflammatory cytokines. Under viral infection, obesity-related chronic inflammation causes reduced macrophage activation and blunts proinflammatory cytokine production upon macrophage stimulation [7]. The reduced macrophage activation by viral infection may explain the poor vaccination response in obese patients. Moreover, B- and T-cell responses are impaired in individuals with obesity with numerical and functional alterations of lymphocytes, and these alterations may increase susceptibility to viral infection. Thus, this dysregulated proinflammatory response contributes to the severe lung lesions seen during the COVID-19 pandemic [6]. Based on this mechanism, individuals with obesity who already have low-level chronic inflammation may be more vulnerable to cytokine storms by COVID-19 infection.

To curb these cytokine storms in COVID-19 patients, anti-inflammatory treatment may be beneficial. However, the use of anti-inflammatory treatments can be a doubleedged sword. Anti-inflammatory medications, such as corticosteroids, may delay the elimination of the virus and increase the risk of secondary infection, especially in those with impaired immune systems. Some proinflammatory cytokine antagonists (for example, IL-6 antagonists) can only inhibit specific inflammatory factors and, thus, may eliminate adverse effects of cytokine storms without preventing the effects of other inflammatory cytokines in removing SARS-CoV-2 from the infected organs. A recent study showed that colchicine treatment had a beneficial effect in adults with obesity and metabolic syndrome in reducing IL-6 [8], which may translate to a beneficial effect in people with obesity and COVID-19 infection. However, 
anti-inflammatory medications, such as Janus kinase inhibitors, which were recently reported to treat COVID-19 patients, can inhibit a variety of inflammatory cytokines including interferon- $\alpha$, which plays an important role in suppressing virus activity, and, thus, may not be suitable for treatment of inflammatory cytokine storms caused by COVID-19 [9].

Given the viral nature of cytokine storms and the substantial impairment of immune systems in severe cases, it is critical to strike a balance between up- and downregulation of inflammatory markers for immune homeostasis. In addition, starting anti-inflammatory treatment at the right time is of pivotal importance and should be tailored in individual patients to achieve the most favorable effects.

The combined use of anti-inflammatory and antiviral drugs may be applied as well. As we mentioned above, some anti-inflammatory therapies may increase viral replication. On the other hand, antiviral treatment to inhibit SARS-CoV-2 replication and block SARS-CoV-2 infection may induce proinflammatory cytokine production [10]. Therefore, additional large-cohort studies are required to substantiate or dismiss this possibility before applying clinical trials.

There are a few studies suggesting that individuals with obesity may be at higher risk of poor outcomes from COVID-19 by hyperinflammation. The inflammatory response may be uncontrolled due to malfunctioning or exhausted immune cells, such as B and T cells and macrophages, in those with obesity and low-level chronic inflammation [7]. Therefore, further large-scale studies are needed to confirm the role of obesity-induced inflammation in the pathogenesis of COVID-19. In this context, a wide variety of strategies such as increased vigilance, early detection and testing, and aggressive treatment should be established for patients with obesity and COVID-19 infection.

Acknowledgements This work was supported by Basic Science Research Program through the National Research Foundation of Korea (NRF) funded by the Ministry of Science, ICT \& Future Planning (NRF2015M3A9B5030157), and "Leaders INdustry-university Cooperation+" Project, supported by the Ministry of Education and National Research Foundation of Korea.

\section{Compliance with ethical standards}

Conflict of interest The authors declare that they have no conflict of interest.

Publisher's note Springer Nature remains neutral with regard to jurisdictional claims in published maps and institutional affiliations.

\section{References}

1. Chiappetta S, Sharma AM, Bottino V, Stier C. Brief communication: COVID-19 and the role of chronic inflammation in patients with obesity. Int J Obes. 2020. https://doi.org/10.1038/ s41366-020-0597-4.

2. Kim J, Na H, Kim J-A, Nam J-H. What we know and what we need to know about adenovirus 36-induced obesity. Int J Obes. 2020. https://doi.org/10.1038/s41366-020-0536-4.

3. Wan S, Yi Q, Fan S, Lv J, Zhang X, Guo L, et al. Characteristics of lymphocyte subsets and cytokines in peripheral blood of 123 hospitalized patients with 2019 novel coronavirus pneumonia (NCP). MedRxiv. 2020. https://doi.org/10.1101/2020.02.10. 20021832.

4. Huang C, Wang Y, Li X, Ren L, Zhao J, Hu Y, et al. Clinical features of patients infected with 2019 novel coronavirus in Wuhan, China. Lancet. 2020;395:497-506.

5. Xu Z, Shi L, Wang Y, Zhang J, Huang L, Zhang C, et al. Pathological findings of COVID-19 associated with acute respiratory distress syndrome. Lancet Respir Med. 2020;8:420-2.

6. Luzi L, Radaelli MG. Influenza and obesity: its odd relationship and the lessons for COVID-19 pandemic. Acta Diabetol. 2020;57:759-64.

7. Ahn S-Y, Sohn S-H, Lee S-Y, Park H-L, Park Y-W, Kim H, et al. The effect of lipopolysaccharide-induced obesity and its chronic inflammation on influenza virus-related pathology. Environ Toxicol Pharmacol. 2015;40:924-30.

8. Demidowich AP, Levine JA, Apps R, Cheung FK, Chen J, Fantoni $\mathrm{G}$, et al. Colchicine's effects on metabolic and inflammatory molecules in adults with obesity and metabolic syndrome: results from a pilot randomized controlled trial. Int J Obes. 2020. (In press).

9. Zhang W, Zhao Y, Zhang F, Wang Q, Li T, Liu Z, et al. The use of anti-inflammatory drugs in the treatment of people with severe coronavirus disease 2019 (COVID-19): the experience of clinical immunologists from China. Clinical Immunology. 2020. https:// doi.org/10.1016/j.clim.2020.108393.

10. Runfeng L, Yunlong H, Jicheng H, Weiqi P, Qinhai M, Yongxia $\mathrm{S}$, et al. Lianhuaqingwen exerts anti-viral and anti-inflammatory activity against novel coronavirus (SARS-CoV-2). Pharmacol Res. 2020. https://doi.org/10.1016/j.phrs.2020.104761. 